Ewa Sylwestrzak (Zielona Góra)

\title{
ON AN INVERSE PROBLEM IN THE THEORY OF THERMISTORS
}

Abstract. An inverse problem for a nonlocal problem describing the temperature of a conducting device is studied.

1. Introduction. In this paper we consider the following nonlocal problem for a nonlinear differential equation:

$$
\begin{gathered}
\theta^{\prime \prime}+\lambda \frac{f(\theta)}{\left(\int_{-1}^{1} f(\theta(x)) d x\right)^{2}}=0, \quad-1<x<1, \\
\theta(-1)=\theta(1)=0,
\end{gathered}
$$

where $\theta:[-1,1] \rightarrow[0, \infty)$ is an unknown function, $f:[0, \infty) \rightarrow(0, \infty)$ is a given function, and $\lambda>0$ is a real parameter. This problem models the stationary temperature $\theta$ of a conducting device occupying the interval $-1 \leq x \leq 1$ when the electric current flows through the material with temperature-dependent electrical resistivity $f(\theta)>0$, subject to a fixed potential difference $V$, and $\lambda=V^{2}$.

The temporal evolution of the temperature $\theta$ and the electric potential $\varphi$ is described by the system

$$
\begin{gathered}
\theta_{t}=\theta_{x x}+\sigma \varphi_{x}^{2}, \\
\left(\sigma \varphi_{x}\right)_{x}=0,
\end{gathered}
$$

where $\sigma(\theta)=1 / f(\theta)$ is the electrical conductivity (see [4]). We assume that the thermal conductivity, the density and the specific heat of the onedimensional conductor are equal to one.

We fix the electric potential at the ends of the conductor, i.e. $\varphi(-1)=V_{1}$, $\varphi(1)=V_{2}$, and we assume that $\theta=0$ at $x= \pm 1$.

2000 Mathematics Subject Classification: 34K29, 34B15.

Key words and phrases: theory of thermistors, inverse problem, existence of solutions.

The preparation of this paper was supported by the KBN grant 2/P03A/011/19. 
From (4) we have $\sigma \varphi_{x}=J(t)$, so

$$
\varphi_{x}=f(\theta) J(t) .
$$

Integrating (5) over $[-1,1]$ we get $J(t)=V / \int_{-1}^{1} f(\theta)$, where $V=V_{2}-V_{1}$. Then we have

$$
\varphi_{x}=V \frac{f(\theta)}{\int_{-1}^{1} f(\theta)} .
$$

Hence we can write the equation (3) in the form

$$
\theta_{t}=\theta_{x x}+\lambda \frac{f(\theta)}{\left(\int_{-1}^{1} f(\theta)\right)^{2}},
$$

where $\lambda=V^{2}$.

Thus the stationary temperature $\theta$ satisfies the equation (1) with the boundary conditions (2).

2. Existence of solutions. Let $\theta_{\lambda}$ be a solution of the problem (1)-(2).

It is known that the solution of (1)-(2) is an even function and the maximum of $\theta_{\lambda}$ is attained at $0, \theta_{\lambda}(0) \equiv M(\lambda)$ (see [2]).

Note that

$$
\lambda=8 \int_{0}^{M(\lambda)} f(s) d s .
$$

Indeed, integrating (1) over $[-1,1]$ and using $\theta_{\lambda}^{\prime}(1)=-\theta_{\lambda}^{\prime}(-1)$ we obtain

$$
2 \theta_{\lambda}^{\prime}(1)+\frac{\lambda}{\int_{-1}^{1} f\left(\theta_{\lambda}\right)}=0
$$

On the other hand, multiplying (1) by $2 \theta_{\lambda}^{\prime}$ and then integrating over $[0,1]$ we get

$$
\left(\theta_{\lambda}^{\prime}(1)\right)^{2}+\frac{2 \lambda}{\left(\int_{-1}^{1} f\left(\theta_{\lambda}\right)\right)^{2}} \int_{0}^{1} f\left(\theta_{\lambda}\right) \theta_{\lambda}^{\prime}=0 .
$$

Hence

$$
\left(\theta_{\lambda}^{\prime}(1)\right)^{2}-\frac{2 \lambda}{\left(\int_{-1}^{1} f\left(\theta_{\lambda}\right)\right)^{2}} \int_{0}^{M(\lambda)} f(s) d s=0 .
$$

Combining (7) and (8) we finally obtain the formula (6).

Hence the maximum $M(\lambda)$ of the solution of the problem (1)-(2) is an increasing function of $\lambda$. Moreover, in the case $\int_{0}^{\infty} f(s) d s=\infty$, we have $M(\lambda) \rightarrow \infty$ as $\lambda \rightarrow \infty$, and if $\int_{0}^{\infty} f(s) d s<\infty$, then $M(\lambda) \rightarrow \infty$ as $\lambda \rightarrow 8 \int_{0}^{\infty} f(s) d s$ (see [5]). 
Under the assumption that $f$ is a positive decreasing function, the existence and uniqueness of solutions of (1)-(2) have been investigated in [2] and [5]. The following facts were proved:

(a) If $\int_{0}^{\infty} f(s) d s=\infty$, then there is a unique solution of the problem (1)-(2) for each $\lambda>0$,

(b) If $\int_{0}^{\infty} f(s) d s<\infty$, then the problem (1)-(2) has

(i) a unique solution for each $\lambda<8 \int_{0}^{\infty} f(s) d s$,

(ii) no solution for $\lambda \geq 8 \int_{0}^{\infty} f(s) d s$.

In [2] the following result was obtained. If $f(\theta)$ is a positive increasing function such that $\theta f^{\prime}(\theta) / f(\theta) \rightarrow \infty$ as $\theta \rightarrow \infty$, then the problem (1)-(2) has a solution for each $\lambda>0$.

Here we generalize the above result proving the existence of a solution of the problem (1)-(2) for an arbitrary positive increasing function $f$.

TheOREM 1. Let $f$ be a continuous positive increasing function. Then there is a solution $\theta$ in $C^{0}[-1,1] \cap C^{2}(-1,1)$ of the problem (1)-(2) for each $\lambda>0$.

Proof. We introduce the following operator $T$ on the space $C^{0}[-1,1]$ :

$$
T \theta(x)=\frac{\lambda}{\left(\int_{-1}^{1} f(|\theta|)\right)^{2}} \int_{-1}^{1} G(x, y) f(|\theta(y)|) d y,
$$

where

$$
G(x, y)= \begin{cases}(1-x)(y+1) / 2 & \text { for }-1 \leq y \leq x \leq 1 \\ (1-y)(x+1) / 2 & \text { for }-1 \leq x \leq y \leq 1\end{cases}
$$

is the Green function for the operator $-\theta^{\prime \prime}$ (see [1]). The solution of the problem (1)-(2) is a fixed point of the map $T$.

The proof of the existence of a fixed point will be based on the LeraySchauder Theorem applied in the space $C^{0}[-1,1]$ with the supremum norm $\|\theta\|_{\infty}$.

The operator $T$ is continuous on $C^{0}[-1,1]$. Moreover $(T \theta(x))^{\prime}$ is uniformly bounded if $\theta$ belongs to a bounded subset of $C^{0}[-1,1]$. Indeed, if $\|\theta\|_{\infty} \leq C$, then $\left\|(T \theta)^{\prime}\right\|_{\infty} \leq \lambda f(C) /\left(4 f^{2}(0)\right)$. Hence $T$ is a compact operator.

To apply the Leray-Schauder Theorem, it is sufficient to prove a uniform a priori estimate of solutions $\theta^{\alpha}$ of the equation $\theta=\alpha T \theta$ for $\alpha \in[0,1]$.

Obviously we have $\left\|\theta^{\alpha}\right\|_{\infty} \leq \lambda /(4 f(0)) \equiv C$ for $\alpha \in[0,1]$.

3. The inverse problem. Here we study an inverse problem related to (1)-(2), consisting in the unique identification of the function $f$ in the case when we have some information about the solutions of the problem (1)-(2). 
First we investigate the problem of the determination of $f$ by the values of the solutions of $(1)-(2)$ on $[0,1]$.

TheOREm 2. The solution $\theta_{\lambda}$ of the problem (1)-(2) uniquely determines the function $f$ on the interval $[0, M(\lambda)]$.

Proof. From (1) we have

$$
f\left(\theta_{\lambda}(x)\right)=-\frac{4\left(\int_{0}^{1} f\left(\theta_{\lambda}\right)\right)^{2}}{\lambda} \theta_{\lambda}^{\prime \prime}(x) .
$$

Integrating (1) over $[0,1]$ we get $\int_{0}^{1} f\left(\theta_{\lambda}\right)=-\lambda /\left(4 \theta_{\lambda}^{\prime}(1)\right)$. Then

$$
f\left(\theta_{\lambda}(x)\right)=-\frac{\lambda \theta_{\lambda}^{\prime \prime}(x)}{4\left(\theta_{\lambda}^{\prime}(1)\right)^{2}}
$$

Note that the solution of $(1)-(2)$ is one-to-one on $[0,1]$. Hence

$$
f(s)=-\frac{\lambda \theta_{\lambda}^{\prime \prime}\left(\theta_{\lambda}^{-1}(s)\right)}{4\left(\theta_{\lambda}^{\prime}(1)\right)^{2}} \quad \text { for } s \in[0, M(\lambda)] .
$$

Corollary 1. Let $\int_{0}^{\infty} f(s) d s=\int_{0}^{\infty} \bar{f}(s) d s=\infty$. If $\theta_{\lambda_{n}}=\bar{\theta}_{\lambda_{n}}$ for some increasing sequence $\lambda_{n}$ tending to $\infty$, then $f=\bar{f}$ on the whole half-line $[0, \infty)$.

Corollary 2. Let $\int_{0}^{\infty} f(s) d s=\int_{0}^{\infty} \bar{f}(s) d s \equiv \lambda^{*}<\infty$. If $\theta_{\lambda_{n}}=\bar{\theta}_{\lambda_{n}}$ for some increasing sequence $\lambda_{n}$ tending to $8 \lambda^{*}$, then $f=\bar{f}$ on the whole half-line $[0, \infty)$.

The formula (9) involves the first and second derivatives of $\theta_{\lambda}$. Therefore small $C^{0}$-changes of $\theta_{\lambda}$ may give big changes in $f$. This kind of instability is typical for inverse problems. Our aim is to describe a procedure for finding $f$ which is stable under $C^{0}$-perturbations of $\theta_{\lambda}$.

We start with the following example. In [3] the authors assumed that the function $f$ has the form $f(\theta)=a e^{b \theta}$, where $a>0, b>0$ are real parameters. In that case the problem (1)-(2) has a unique solution for all $\lambda>0$ (see [2]). Our aim is to determine $a$ and $b$ if we know the value of the temperature $\theta_{\lambda}(0)=M(\lambda)$ for some $\lambda=\lambda_{j}, j=1,2$. The formula (6) takes the form $\lambda / 8=a\left(e^{b M(\lambda)}-1\right) / b$, and we obtain $a=\lambda_{1} b /\left(8\left(e^{b M\left(\lambda_{1}\right)}-1\right)\right)$ and $\lambda_{1} / \lambda_{2}=\left(e^{b M\left(\lambda_{1}\right)}-1\right) /\left(e^{b M\left(\lambda_{2}\right)}-1\right)$. Let $\lambda_{1}>\lambda_{2}$. Then $M\left(\lambda_{1}\right)>M\left(\lambda_{2}\right)$ and we note that the right hand side of the last equation is an increasing function of $b$. Hence we can determine uniquely the parameters $a, b$, provided $\lambda_{1} / \lambda_{2}>M\left(\lambda_{1}\right) / M\left(\lambda_{2}\right)$.

On the other hand, if $\lambda_{1} / \lambda_{2} \leq M\left(\lambda_{1}\right) / M\left(\lambda_{2}\right)$ for some $\lambda_{1}, \lambda_{2}$, we can conclude that the dependence of the resistivity of the thermistor on the temperature is not given by a function of the form $f(\theta)=a e^{b \theta}$. 
We prove that $M(\lambda)$ determines $f$ not only for $f$ of the special form $f(\theta)=a e^{b \theta}$ but also for arbitrary $f$. Assume that $M(\lambda)$ is a differentiable function of $\lambda$. Then using the formula (6) we get the relation $f(M(\lambda))=$ $\left(8 M^{\prime}(\lambda)\right)^{-1}$. Hence $f$ is uniquely determined by $M$. We see that small changes of $M(\lambda)$ in $C^{0}$-norm may give big changes of $f$. In practice the values $M(\lambda)$ are obtained experimentally, hence it is reasonable to ask about the influence of inaccuracy in measuring $M(\lambda)$ on the function $f$. Below we show that under some assumptions on the dependence of the resistivity $f$ on the temperature $\theta$, small $C^{0}$-perturbations of $M$ lead to small changes of $f$.

TheOREM 3. Let $f_{n}, f$ be differentiable positive functions such that the sequences $f_{n}, f^{\prime}-f_{n}^{\prime}$ are uniformly bounded on $[0, \infty)$, and suppose $\int_{0}^{\infty} f(s) d s=\int_{0}^{\infty} f_{n}(s) d s=\infty$. Assume that the sequence $M_{n}(\lambda)$ converges uniformly to $M(\lambda)$ on $[0, \bar{\lambda}]$. Then the sequence $f_{n}(s)$ tends uniformly to $f(s)$ on $[0, M(\bar{\lambda})]$.

Proof. Let $f_{n} \leq c$ for some constant $c>0$.

From (6) we get $\int_{0}^{M(\lambda)} f(s) d s=\int_{0}^{M_{n}(\lambda)} f_{n}(s) d s$. Thus

$$
\left|\int_{0}^{M(\lambda)}\left(f(s)-f_{n}(s)\right) d s\right|=\left|\int_{M(\lambda)}^{M_{n}(\lambda)} f_{n}(s) d s\right| \leq c\left|M_{n}(\lambda)-M(\lambda)\right| .
$$

Let $g_{n}(x)=\int_{0}^{x} h_{n}(s) d s$, where $x \equiv M(\lambda)$ and $h_{n} \equiv f-f_{n}$. Then by the assumption of the theorem it follows from (10) that the sequence $g_{n}$ tends uniformly to 0 on $[0, M(\bar{\lambda})]$.

The sequence $h_{n}^{\prime}$ is uniformly bounded, so by the Arzelà-Ascoli theorem from each subsequence of $h_{n}$ we can choose some subsequence $h_{n_{k}}$ uniformly convergent to some function $h$. We have $g_{n_{k}}(x)=\int_{0}^{x} h_{n_{k}}(s) d s$ and $g_{n_{k}} \rightarrow 0$ uniformly on $[0, M(\bar{\lambda})]$. Hence $\int_{0}^{x} h(s) d s=0$ for each $x>0$, which implies $h=0$, and thus the sequence $h_{n}=f-f_{n}$ tends uniformly to 0 on $[0, M(\bar{\lambda})]$.

REMARK. The assertion of Theorem 3 holds if we consider the differentiable positive functions $f_{n}, f$ such that $f_{n}$ are decreasing, the sequences $f_{n}^{\prime}$, $f^{\prime}-f_{n}^{\prime}$ are uniformly bounded on $[0, \infty)$ and $\int_{0}^{\infty} f(s) d s=\int_{0}^{\infty} f_{n}(s) d s=\infty$.

It is sufficient to prove that the sequence $f_{n}(0)$ is bounded. Suppose that $f_{n}(0) \rightarrow \infty$. Then from the relation $\lambda=8 \int_{0}^{M_{n}(\lambda)} f_{n}(s) d s$ and uniform boundedness of $f_{n}^{\prime}$ it follows that $M_{n}(\lambda)$ tends to 0 for each $\lambda>0$. Moreover the $M_{n}(\lambda)$ are increasing functions of $\lambda$. Thus we get uniform convergence of the sequence $M_{n}(\lambda)$ to $M(\lambda) \equiv 0$, a contradiction.

Immediately from Theorem 3 we get 
Corollary 3. Let $\int_{0}^{\infty} f_{j}(s) d s=\infty, j=1,2$. If $M_{1}(\lambda)=M_{2}(\lambda)$ for each $\lambda>0$, then $f_{1}=f_{2}$ on the whole half-line $[0, \infty)$.

Acknowledgments. The author would like to thank P. Biler and T. Nadzieja for interesting conversations during the preparation of this paper.

\section{References}

[1] P. B. Bailey, L. F. Shampine and P. E. Waltman, Nonlinear Two Point Boundary Value Problems, Academic Press, New York, 1968.

[2] J. W. Bebernes and A. A. Lacey, Global existence and finite-time blow-up for a class of nonlocal parabolic problems, Adv. Differ. Equ. 2 (1997), 927-953.

[3] E. Kuźma and B. Schmidt, Thermistors, Wydawnictwa Naukowo-Techniczne, Warszawa, 1972 (in Polish).

[4] A. A. Lacey, Thermal runaway in a nonlocal problem modelling Ohmic heating. Part I: Model derivation and some special cases, Euro. J. Appl. Math. 6 (1995), 127-144.

[5] -, Thermal runaway in a nonlocal problem modelling Ohmic heating. Part II: General proof of blow-up and asymptotics of runaway, ibid., 201-224.

Institute of Mathematics

University of Zielona Góra

Podgórna 50

65-246 Zielona Góra, Poland

E-mail: e.sylwestrzak@im.uz.zgora.pl

Received on 13.5.2002;

revised version on 30.8.2002 